\title{
Comparison of Incidental Radiation Dose to Axilla and Internal Mammary Nodal Area by Conventional, 3DCRT, and IMRT Technique in Carcinoma of Breast
}

Govardhan HB$^{1^{*}}$, Naveen Thimmaiah ${ }^{1}$, Khaleel IA ${ }^{1}$, Satyajit Pradhan ${ }^{2}$, Rajeev jain ${ }^{3}$, Sridhar $\mathbf{P}^{1}$, Paddad Siddanna ${ }^{1}$, Nabiza Begum $^{1}$, Nivedita $^{1}{ }^{1}$, Lalit $\mathbf{K}^{1}$, Uday $\mathbf{K}^{1}$ and Sham Sunder ${ }^{1}$

${ }^{1}$ Department of Radiation Oncology, Kidwai Memorial Institute of oncology, Bangalore, Karnataka, India

${ }^{2}$ Department of Radiotherapy and Radiation Medicine, IMS, BHU, Varanasi, India

${ }^{3}$ Department of Radiotherapy, Government Medical College, Raipur, Chhattisgarh, India

"Corresponding author: Govardhan HB, Department of Radiation Oncology, Kidwai Memorial Institute of Oncology, Bangalore, Karnataka, 560030, India, Tel: 9971058822; E-mail: govardhanhb@gmail.com

Received date: January 27, 2017; Accepted date: February 04, 2017; Published date: February 10, 2017

Copyright: (c) 2017 Govardhan HB, et al. This is an open-access article distributed under the terms of the Creative Commons Attribution License, which permits unrestricted use, distribution, and reproduction in any medium, provided the original author and source are credited.

\begin{abstract}
Purpose: To quantify the incidental radiation dose delivered to axilla and internal mammary nodal (IMN) area by Conventional Tangential Radiation Therapy (CRT), 3 Dimensional Conformal Radiation Therapy (3DCRT) and Intensity Modulated Radiation Therapy (IMRT).

Methods and Materials: We prospectively evaluated incidental radiation to axilla in twenty cases of breast cancer treated with adjuvant radiation therapy. Three plans were generated for each case, comprising CRT, 3DCRT and IMRT tangents. Radiation doses to axillary levels I, II, III, and IMN areas were evaluated for mean dose, V95, V80 and V50. Comparisons were made using ANOVA.

Results: The mean volume and range of the axillary level I, II, III, and IMN were $61.1 \mathrm{cc}$ and $142-57 \mathrm{cc} ; 42.6 \mathrm{cc}$ and $61-21 \mathrm{cc} ; 19.5 \mathrm{cc}$ and $34-15 \mathrm{cc} ; 13.2 \mathrm{cc}$ and $21-9 \mathrm{cc}$ respectively. The mean dose to axilla by 3 techniques (by IMRT, 3DCRT and CRT) to Level I, II, III, and IMN were $75 \%, 53 \%, 38 \%$, and $61 \%$ vs. $81 \%, 64 \%$, $44 \%$ and $77 \%$ vs. $92 \%, 86 \%, 53 \%$ and $92 \%$ respectively $(p<0.05)$. The V95 values (volume receiving $95 \%$ of dose) for the three techniques were $43 \%, 39 \%, 17 \%$ and $49 \%$ by IMRT: $40 \%, 45 \%, 21 \%$ and $59 \%$ by 3 DCRT; $72 \%, 61 \%, 24 \%$ and $65 \%$ by CRT (IMRT vs. 3DCRT for level II axilla, IMRT vs. CRT and 3DCRT vs. CRT-p<0.05) The V80 were $49 \%, 53 \%$, $29 \%$, and $57 \%$ by IMRT; $55 \%, 47 \%, 34 \%$ and $68 \%$ by 3DCRT; $85 \%, 77 \%, 44 \%$ and $69 \%$ by CT (IMRT vs. 3DCRT for level III axilla and IMN, IMRT vs. CRT and 3DCRT vs. CRT -p<0.05). The V50 values were $75 \%, 65 \%, 41 \%$ and $66 \%$ by IMRT; $82 \%, 53 \%, 57 \%$ and $84 \%$ by 3 DCRT; $94 \%, 89 \%, 42 \%$ and $90 \%$ by CRT (IMRT vs. 3DCRT, IMRT vs. CRT, and 3DCRT vs. CRP-p<0.05).
\end{abstract}

Conclusion: Axillary and internal mammary nodal areas receive substantial amount of incidental radiation doses with all the three techniques; however, conformal techniques (IMRT, 3DCRT) deliver significantly lesser incidental radiation to lower axilla than CRT technique.

Keywords: Breast cancer; Axilla; Internal mammary node; Incidental dose

\section{Introduction:}

Breast cancer is one of the common cancers in females worldwide. These days standard practice is breast conserving surgery followed by adjuvant radiotherapy for early presentation [1] and mastectomy followed by adjuvant radiotherapy of the chest wall with or without chemotherapy in advanced cases [2]. Many studies [3,4] have shown that adjuvant radiotherapy to the chest wall improves local control and also survival in node positive and advanced node negative breast cancer patients after mastectomy. The adjuvant radiotherapy of the chest wall is commonly achieved with tangential beams. They include part of the anterior thoracic cavity which in turn leads to significant radiation to heart and lung leading to the risk of long-term pulmonary and cardiac complications [5]. The conformal radiation therapy like three-dimensional conformal radiotherapy (3DCRT) and intensity modulated radiotherapy (IMRT) leads to favorable dose distribution and less dose to these critical organs [6,7]. All above data are basically on breast conservative cases. There is still scarce literature on dose distribution by all three radiation techniques in post mastectomy breast carcinoma [8,9]. In breast cancer pT1-3 with 1 to 3 positive lymph nodes after completed axillary dissection, external beam radiation to breast alone is standard of care [10]. Patients not receiving radiation even to the breast have more frequent axillary recurrence [11]. Early breast cancer with positive sentinel-node have about $40 \%$ chances of having additional nodes on complete axillary dissection and also add significance chance of internal mammary chain lymph nodes. In a trial conducted by American College of Surgeons Oncology Group (Z0011), randomized patients of early breast cancer who underwent breast conservation surgery and had positive sentinel node biopsy (SNB) to standard axillary dissection or no further axillary surgery, followed by radiation to breast alone in both the arms. The study concluded that axillary failures were same in both the arms [12] raising the possibility that incidental dose of radiation to axilla when breast is being irradiated might contribute in eradicating microscopic disease in undissected axilla. 
Citation: Govardhan HB, Thimmaiah N, Khaleel IA, Pradhan S, jain R, et al. (2017) Comparison of Incidental Radiation Dose to Axilla and Internal Mammary Nodal Area by Conventional, 3DCRT, and IMRT Technique in Carcinoma of Breast. J Nucl Med Radiat Ther 8: 325. doi:10.4172/2155-9619.1000325

Page 2 of 6

Whenever in early case of Breast carcinoma where lymph node irradiation is not indicated or not needed, then the question definitely arises on incidental radiation dose to these area on over treatment that help to treat micro-metastasis or this may attribute to extra complications in term of lymphedema. Axillary lymph node dissection can lead to some of the most debilitating morbidity for breast cancer patients which include arm lymphedema, shoulder mobility impairment, and brachial plexopathy in $20 \%$ of cases which leads to impairment in quality of life (QOL) [13]. This is greatly enhanced by the combination of axillary surgery and radiation to approximately $40 \%$ [14]. Thus this contributory radiation from chest wall radiation to axilla may raise a question of benefit vs. complication when radiation is intended for breast or chest wall irradiation alone.

In the same way, the incidental radiation dose to internal mammary group of lymph node in those cases may help to combat the micrometastasis is still not clear. Again, unexpected radiation doses to this area raise a question of benefit vs. complication in breast or chest wall irradiation alone. There are some investigators who have analyzed the coverage of axillary region. No literature present suggestive of incidental radiation dose to internal mammary nodal region in any of the radiation technique. Also literature is sparse on incidental doses especially with newer techniques. Thus, we are aiming to know the difference in technique in terms of incidental radiation dose to these areas and possible clinical relevance.

This study attempts to quantify the incidental dose delivered to axilla and internal mammary area by three techniques (conventional tangential, 3DCRT and IMRT), which might help us understand the role of incidental radiation in controlling microscopic disease and/or increasing the incidence of axillary toxicity.

\section{Materials and Methods}

This study was conducted in three regional cancer centers. We prospectively evaluated twenty cases of breast cancers that underwent either breast conservative surgery (10 patients) or modified radical mastectomy (10 cases). The patients who were planned for adjuvant radiation therapy were taken for the study. All these patients were treated with IMRT/3DCRT to whole breast or chest wall. The ipsilateral axillary nodal levels and ipsilateral internal mammary node levels were delineated. The incidental doses were dosimetrically compared in these regions by all three techniques

Simulation: CT simulations were done on supine position with hands above head. Immobilization done by thermoplastic thoracic cast. Radio-opaque markers were placed to delineate the extent of clinically palpable breast tissue in BCS cases and in post mastectomy cases at the second intercostal space or opposite breast level whichever was superior, for inferior extent $2 \mathrm{~cm}$ below infra mammary folds of opposite breast level, and at mid axillary line laterally, mid line medially. A set of 3 fiducials was used to ascertain the positioning and setup. Patients were scanned from the lower edge of mandible to mid abdomen with $3 \mathrm{~mm}$ slice thickness and slice separation.
Contouring: Delineation of target, axillary volumes and organ at risk was done on Eclipse Varian system version 8 work stations. Clinical target volume (CTV) and Ipsilateral Axilla (Level I, II, III) in both breast conservatory cases and in post mastectomy cases contoured as per RTOG (Radiation Therapy and Oncology Group) breast cancer atlas for radiation planning. Consensus definition [15] aided by the markers placed during simulation. Planning target volume (PTV) was constructed by expanding CTV by $10 \mathrm{~mm}$ all around except skin where $3 \mathrm{~mm}$ is taken. The critical structures delineated were lungs, heart, contralateral breast, ipsilateral humeral head, trachea, and esophagus. Ipsilateral internal mammary group of lymph node were drawn by contouring ipsilateral internal mammary vessels from $1^{\text {st }}$ to $4^{\text {th }}$ intercostal spaces and a safety margin of $7 \mathrm{~mm}$ was given.

Dose prescription: Dose prescribed to PTV was 50 Gy in 25 fractions at $95 \%$ coverage volume getting at least $95 \%$ of the prescribed dose. Three plans were devised for each CT data set by Eclipse version 8 varian planning system which includes standard conventional tangent with half beam block, 3D conformal Radiation therapy (3DCRT) plan and tangential intensity modulated radiation therapy (IMRT) plan. For the conventional tangent plan, the superior and inferior border of the conventional opposed tangential irradiation fields were placed at superior and inferior fiducial level.

Efforts were made to keep the amount of lung as measured in the tangential field from deep edge to chest (mean lung distance MLD) wall within $1.5 \mathrm{~cm}$ to $2.5 \mathrm{~cm}$. Superposition algorithm was used for dose calculation in all treatment plans. For 3DCRT and IMRT plans, the goal was coverage of at least $95 \%$ of PTV with $95 \%$ of prescribed dose. The physics team was blinded to the axillary contours. Plan evaluation and statistical analysis: Dose volume histograms (DVH) were used to evaluate all the three plans of each case with respect to PTV coverage and OAR sparing.

Doses to axillary and internal mammary nodal levels were compared between the three plans using DVH. Axillary level I, II, III, and internal mammary group were evaluated for mean dose, volume receiving $95 \%$ of prescribed dose (V95\%), V80\% and V50\%. Analysis was carried out using SPSS version 16 and comparison was made using ANOVA. Difference was regarded statistically significant at $p$ value of $<0.05$.

\section{Results:}

A total of twenty patients were selected. Mean age of the patient is $58 \pm 13$ years. All patients were infiltrative breast cancer (the details are given in Table 1). The mean volume and range of the axillary level 1, II, III, and internal mammary chain were $61.1 \mathrm{cc}$ and $142-57 \mathrm{cc} ; 42.6 \mathrm{cc}$ and $61-21 \mathrm{cc} ; 19.5 \mathrm{cc}$ and $34-15 \mathrm{cc} ; 13.2 \mathrm{cc}$ and $21-9 \mathrm{cc}$, respectively. The D95, D80, D50 of axillary level I, II, III and internal mammary area that receive by all the techniques is given in Table 2 .

\begin{tabular}{|l|l|}
\hline Characteristic & Patients $\mathbf{( N = 2 0 )}$ \\
\hline Age (Years) & $58 \pm 13$ years \\
\hline Laterality-Right & $12(60 \%)$ \\
\hline Laterality-Left & $8(40 \%)$ \\
\hline
\end{tabular}


Citation: Govardhan HB, Thimmaiah N, Khaleel IA, Pradhan S, jain R, et al. (2017) Comparison of Incidental Radiation Dose to Axilla and Internal Mammary Nodal Area by Conventional, 3DCRT, and IMRT Technique in Carcinoma of Breast. J Nucl Med Radiat Ther 8: 325. doi:10.4172/2155-9619.1000325

Page 3 of 6

\begin{tabular}{|l|l|l|}
\hline $\begin{array}{l}\text { Histopathology-Infiltrative } \\
\text { ductal carcinoma (IDC) }\end{array}$ & \multicolumn{2}{l|}{$20(100 \%)$} \\
\hline Stage of tumor & $6(30 \%)$ \\
\hline T1 & $5(25 \%)$ \\
\hline T2 & $7(35 \%)$ \\
\hline T3 & $2(10 \%)$ \\
\hline T4 & \multicolumn{2}{|l|}{} \\
\hline Node & $12(60 \%)$ \\
\hline No & $8(40)$ \\
\hline N1 & \\
\hline
\end{tabular}

Table 1: Patient characteristics.

\begin{tabular}{|c|c|c|c|c|c|c|c|c|c|}
\hline \multirow{2}{*}{$\begin{array}{l}\text { Nodal } \\
\text { stations }\end{array}$} & \multicolumn{3}{|c|}{ D95 (\%) } & \multicolumn{3}{|c|}{ D80 (\%) } & \multicolumn{3}{|c|}{ D50 (\%) } \\
\hline & IMRT & 3DCRT & Conv & IMRT & 3DCRT & Conv & IMRT & 3DCRT & Conv \\
\hline Axilla L-1 & 42 & 36 & 61 & 53 & 56 & 67 & 72 & 77 & 89 \\
\hline Axilla L-2 & 33 & 41 & 54 & 43 & 44 & 59 & 55 & 54 & 85 \\
\hline Axilla L-3 & 17 & 21 & 41 & 35 & 53 & 47 & 67 & 75 & 61 \\
\hline IMC & 22 & 25 & 63 & 53 & 57 & 69 & 63 & 76 & 90 \\
\hline
\end{tabular}

Table 2: Incidental radiation dose (\%) of the nodal levels that received by significant volume.

The average percent mean dose delivered to axillary level I, II, III and intra-mammary chain by 3 different techniques is shown in Table 3. The mean dose to axilla by 3 techniques (by IMRT, 3DCRT and CT) to Level 1, II, III, and IMN were $75 \%, 53 \%, 38 \%$, and $61 \%$ vs. $81 \%$, $64 \%, 44 \%$ and $77 \%$ vs. $92 \%, 86 \%, 53 \%$ and $92 \%$ respectively ( $\mathrm{p}<0.05)$.

\begin{tabular}{|l|l|l|l|l|}
\hline Nodal stations & IMRT (\%) & 3DCRT (\%) & Conventional (\%) & P \\
\hline Axilla Level 1 & 75 & 81 & 92 & 0.01 \\
\hline Axilla Level 2 & 53 & 64 & 86 & 0 \\
\hline Axilla Level 3 & 38 & 44 & 53 & 0.001 \\
\hline IMN & 61 & 77 & 92 & 0.004 \\
\hline
\end{tabular}

Table 3: The average percent mean dose delivered to axilla and internal mammary nodal (IMN) area.

The V95 values (volume receiving $95 \%$ of dose) for the three techniques were $43 \%, 39 \%, 17 \%$, and $49 \%$ by IMRT; $40 \%, 45 \%, 21 \%$ and $59 \%$ by 3 DCRT; $72 \%, 61 \%, 24 \%$ and $65 \%$ by CRT (IMRT vs. 3DCRT $\mathrm{p}<0.05$ for level II axilla, IMRT vs. CRT and 3DCRT vs. CRT $\mathrm{p}$ value is significant for all nodal area). The V80 were $49 \%$, 53\%, $29 \%$ and $57 \%$ by IMRT; $55 \%, 47 \%, 34 \%$ and $68 \%$ by 3 DCRT; $85 \%, 77 \%$,
$44 \%$ and $69 \%$ by CRT (IMRT vs. 3DCRT $\mathrm{p}<0.05$ for level III axilla and IMN, IMRT vs. CRT and 3DCRT vs. CRT p value is significant for all nodal area). The V50 values were $75 \%, 65 \%, 41 \%$ and $66 \%$ by IMRT; $82 \%, 53 \%, 57 \%$ and $84 \%$ by 3DCRT; $94 \%, 89 \%, 42 \%$ and $90 \%$ by CRT (IMRT vs. 3DCRT, IMRT vs. CRT and 3DCRT vs. CRT $\mathrm{p}$ value is significant for all nodal areas) as given in Tables 4 and 5 .

\begin{tabular}{|l|l|l|l|l|l|l|l|l|l|}
\hline Nodal & \multicolumn{3}{|l|}{ V95 (\%) } & \multicolumn{3}{l|}{ V80 (\%) } & \multicolumn{3}{l|}{ V50 (\%) } \\
\hline stations & IMRT & 3DCRT & Conv & IMRT & 3DCRT & Conv & IMRT & 3DCRT & Conv \\
\hline Axilla L- 1 & 43 & 40 & 72 & 49 & 55 & 85 & 75 & 82 & 94 \\
\hline
\end{tabular}


Citation: Govardhan HB, Thimmaiah N, Khaleel IA, Pradhan S, jain R, et al. (2017) Comparison of Incidental Radiation Dose to Axilla and Internal Mammary Nodal Area by Conventional, 3DCRT, and IMRT Technique in Carcinoma of Breast. J Nucl Med Radiat Ther 8: 325. doi:10.4172/2155-9619.1000325

Page 4 of 6

\begin{tabular}{|l|l|l|l|l|l|l|l|l|l|}
\hline Axilla L- 2 & 39 & 45 & 61 & 53 & 47 & 77 & 65 & 53 & 89 \\
\hline Axilla L- 3 & 17 & 21 & 24 & 29 & 34 & 44 & 41 & 57 & 42 \\
\hline IMN & 49 & 59 & 65 & 57 & 68 & 69 & 66 & 84 & 90 \\
\hline
\end{tabular}

Table 4: Volume (\%) of the nodal levels that receive significant incidental radiation dose.

\begin{tabular}{|c|c|c|c|c|c|c|c|c|c|c|c|c|}
\hline \multirow{2}{*}{$P$ values } & \multicolumn{4}{|c|}{ V95 } & \multicolumn{5}{|c|}{ V80 } & \multicolumn{3}{|c|}{ V50 } \\
\hline & I & II & III & IMC & 1 & II & III & IMC & 1 & II & III & IMC \\
\hline IMRT vs. 3DCRT & 0.3 & 0.09 & 0.21 & 0.43 & 0.53 & 0.52 & 0.03 & 0.09 & 0.01 & 0.24 & 0.04 & 0.01 \\
\hline IMRT vs. Conv & 0.02 & 0.01 & 0.01 & 0.02 & 0.02 & 0.01 & 0 & 0.01 & 0 & 0.03 & 0.51 & 0 \\
\hline 3DCRT vs. Conv & 0.01 & 0.02 & 0.02 & 0.01 & 0.01 & 0.04 & 0.3 & 0.01 & 0.01 & 0 & 0.41 & 0.03 \\
\hline
\end{tabular}

Table 5: Comparison of different groups.

\section{Discussion}

With the widespread use of mammographic screening and breast self-examination, proportion of cases being detected with early staged breast cancer is reduced [16] and thus the role of radiation to internal mammary and axillary node is reduced. In SNB (Sentinal Nodal Biopsy) positive cases ( $<3$ nodes), axillary dissection of level I, II followed by Radiation therapy (RT) to breast or chest wall, supraclavicular region, infraclavicular region is indicated whereas negative SNB cases are treated by RT to breast or chest wall alone. In locally advanced cases, axillary RT is given when axillary dissection is inadequate, more than 3 axillary nodes are positive or extracapsular spread is detected. The internal mammary nodes are included in stage III or radiaologically positive nodes or axillary node positive with inner quadrant tumors. Postoperative radiotherapy is conventionally delivered with standard tangential fields with beam splitter. However, with the introduction of conformal and intensity modulated techniques, tumor control can be achieved with improved dose homogeneity in target volume and reduced dose to organ at risk [17].

Many studies have shown there will be better long term axillary control with breast irradiation and higher nodal recurrence when breast irradiation is skipped $[11,12]$. That can have direct correlation, with standard tangents, the axilla receives a significant incidental dose but still adequate for clinical control of disease is unknown [18,19]. Substantial variability was observed in contouring the targets and organ at risk between observers and the structure overlaps were as low as $10 \%$ and volume variation had standard variation up to $60 \%$ [20]. The average volume of axillary level I, II, III as observed by Julia in 35 patients was $85 \mathrm{cc}, 17 \mathrm{cc}, 5 \mathrm{cc}$ whereas the mean volume of the axillary level I was $28.9 \mathrm{cc}$ (range 14.5 to $58.6 \mathrm{cc}$ ) in a study by Orecchia et al. [21] in 15 patients.

In our study, the mean volume and range of the axillary level 1, II, III and internal mammary chain were $61.1 \mathrm{cc}$ and 142-57 cc; $42.6 \mathrm{cc}$ and $61-21 \mathrm{cc} ; 19.5 \mathrm{cc}$ and $34-15 \mathrm{cc} ; 13.2 \mathrm{cc}$ and $21-9 \mathrm{cc}$, respectively using RTOG contouring guidelines. As per our knowledge, no literature is suggestive of guideline for contouring and volume in terms of internal mammary chain.
In a study by Orecchia et al. [21] in early breast cancers treated by BCS (Breast conservative surgery) and RT to breast alone, the mean volume of first axillary level receiving at least $80 \%$ of the prescribed dose was $30.7 \%$, ranging from $0 \%$ to $70 \%$. The mean dose delivered ranged from $5 \%$ to $80 \%$ of prescribed dose with a mean value of $48.7 \%$. In a similar study, Krasin et al. [22] observed that adequate coverage of level I axilla was achieved only in 1 patient, about $25 \%$ of patients had one half of axilla level I covered by an adequate dose, but no patient had adequate coverage of level II, III, and the mean dose to level I, II, III was $32 \mathrm{~Gy}, 26.5 \mathrm{~Gy}, 18.2 \mathrm{~Gy}$ [18]. Median dose administered to level I, II as observed in a case of BCS treated by standard tangent was 38.6 Gy and 20.6 Gy and the median dose to $90 \%$ (D90\%) of both level I, II was $6.75 \mathrm{~Gy}, 1.75 \mathrm{~Gy}$ [22]. With modern techniques, adequate coverage of the axilla using the high tangential field seems to be dependent on cranial field edge/humeral head and the inclusion of $2 \mathrm{~cm}$ to $2.5 \mathrm{~cm}$ of lung in the tangential fields using multileaf collimators. Alço et al. [23] compared classical high tangential fields with a modified technique using multileaf collimators. The doses received by $95 \%$ of axillary level I and II volumes increased from 16.79 and 11.59 Gy to 47.2 Gy and 45.03 Gy with the multileaf technique.

Li et al. [24] observed the average dose delivered to the level I, II, III with standard tangential field was $66 \%, 44 \%$ and $31 \%$ of the prescribed dose. The coverage increased to $86 \%, 71 \%$ and $73 \%$ with high tangential fields. $51 \%$ of level I, $26 \%$ of level II and $15 \%$ of level III received $95 \%$ (V95\%) of the dose and this was increased to $79 \%, 51 \%$, and $49 \%$ for high tangents (20). The average dose delivered to level I, II, III by IMRT technique was $80.7 \%, 69 \%, 52.3 \%$ and by conventional technique it was $85.8 \%, 67 \%$ and $41.8 \%$. By outlining the regions of surgical clips and axillary tissue on CT scans, Smitt et al. [24] observed that only $1 / 6$ patients had the lower axilla treated to $90 \%$ of the prescribed dose with conventional tangents [24]. Goodman et al. [25] contoured the CT scans of 55 patients after SNB and ALND (Axillary Lymph node dissection) and found that majority of patients had level I nodes covered by $90 \%$ isodose line.

In our study, the volume of level I that receive $95 \%$ of prescribed dose was $43 \%, 40 \%$ and $72 \%$; for level II, it was $35.8 \%, 49 \%$ and $61.2 \%$; and for level III, $17 \%, 21 \%, 24 \%$ respectively for IMRT, 3DCRT, and conventional tangents. The V95, V80 and V50 of internal mammary 
Citation: Govardhan HB, Thimmaiah N, Khaleel IA, Pradhan S, jain R, et al. (2017) Comparison of Incidental Radiation Dose to Axilla and Internal Mammary Nodal Area by Conventional, 3DCRT, and IMRT Technique in Carcinoma of Breast. J Nucl Med Radiat Ther 8: 325. doi:10.4172/2155-9619.1000325

Page 5 of 6

node were $49 \%, 59 \%, 65 \%$ vs. $57 \%, 68 \%, 69 \%$ vs. $66 \%, 84 \%, 90 \%$ by IMRT, 3DCRT and conventional tangential techniques respectively. V95\%, V80\% and V50\% a better dose distribution in IMRT compared to 3DCRT and CT, in axilla V80\% values for Level I, II and Level III in 3DCRT is better than IMRT and CT. For internal mammary nodal area, IMRT has low coverage and dose distribution than other two techniques. None of the patients had adequate coverage at any axillary and internal mammary levels, defined as $95 \%$ of volume enclosed by $95 \%$ of prescribed dose levels.

On comparison both IMRT and 3DCRT was found to have statistically significant lower radiation dose to Axilla level I,II,III and IMN in comparison to CRT $(\mathrm{p}<0.05)$. However, on comparing IMRT and 3DCRT, IMRT was found to give statistically significant lower radiation dose at V80 and V50 to Axillary level III and IMN area $(\mathrm{p}<0.05)$.

Literature suggests that tangential radiation do not adequately cover axilla, hence do not therapeutically treat axilla [24] but on the contrary, several randomized trials including NSABP B-06 demonstrate that regional failure decreases when the whole breast is treated $[2,26]$. Why the regional control improves in tangential irradiation is a matter of speculation and debate. It has been demonstrated that among patients with positive SNB $45 \%$ to $55 \%$ were found to have additional node disease on ALND [27]. Trial conducted by American College of Surgeons Oncology Group (Z0011) randomized patients treated with breast conservation surgery and positive sentinel node biopsy (SNB) to standard axillary dissection or no further surgery, followed by radiation to breast alone in both the arms. The study concluded that at a median follow-up time of 6.3 years, there were no statistically significant differences in local recurrence $(\mathrm{P}=0.11)$ or regional recurrence $(\mathrm{P}=0.45)$ between the 2 groups [12]. One possibility is that dose less that $95 \%$ of prescription dose is adequate to treat clinically negative axilla/microscopic disease. Wither et al have proposed a shallow dose response curve for microscopic disease though a dose of $45 \mathrm{~Gy}$ to $50 \mathrm{~Gy}$ seems more relevant for sterilization of subclinical disease [28]. It is possible that a dose in order of $30 \mathrm{~Gy}$ can bring about some regional control. There are also suggestions from literature that worthwhile benefit can be achieved by the dose as low as 14 Gy to 21 Gy, under the condition of being delivered close to the treatment of primary $[29,30]$ which may explain the regional control in Z0011 and other studies with such incidental axillary dose.

In our study, the average mean radiation dose delivered to axilla Level 1, II, III and internal mammary nodes were $75 \%, 53 \%, 38 \%$ and $61 \%$ vs. $81 \%, 64 \%, 44 \%$ and $77 \%$ vs. $92 \%, 86 \%, 53 \%$ and $92 \%$ by IMRT, 3DCRT and conventional method respectively $(\mathrm{p}<0.05)$, so a significant mean dose of $38 \%$ to $92 \%$ is received to the lymphatic area.

Incidental dose to axilla and internal mammary node is decreased with IMRT as compared to conventional tangent and 3DCRT and coverage is not adequate so our results are consistent with previous studies that axilla is not adequately covered with standard tangents $[18,24]$ but in majority of patients, radiation therapy designed to treat breast only will coincidently treat a significant portion of axilla.

Analysis of the literature shows that complication rates of the ALND and axillary RT are in the same range. ALND is responsible for lymphedema of the arm and restriction of shoulder motion in $10 \%$ to $20 \%$ of the patients. The objective arm edema rate is reported in approximately $10 \%$ patients after axillary radiotherapy. The impairment in shoulder function is approximately $17 \%$ [29-31]. SNB in place of ALND decreases morbidity but does not eliminate it as demonstrated by ALMANAC randomized trial comparing quality of life outcomes between SNB alone and standard axillary treatment group (ALND or Axillary RT). The patients receiving only SNB as axillary treatment had lymphedema in $5 \%$ of cases and sensory loss in $11 \%$ cases versus $13 \%$ and $31 \%$ in patients receiving axillary radiation/ ALND [7]. Studies have been conducted on increased pulmonary toxicity as a complication of radiation to internal mammary node. However, there is a lack of radiobiological study on benefit vs. complication on incidental radiation dose to IMN area.

\section{Conclusion}

During radiation to the chest wall, a substantial incidental radiation dose is also delivered to the axilla and internal mammary nodal area which deals with micro metastasis/ isolated tumor cells but, however, leads to additive toxicity. Among the three techniques, incidental mean radiation dose to axilla and internal mammary nodes is low in IMRT followed by 3DCRT and conventional tangential beam, therefore before starting the treatment, the clinical benefits of the incidental radiation versus the limitation of the toxicity have to be taken into consideration. Based on this study we propose that in cases where conformal radiation technique is used in low risk axillary nodes, constraints to the axillary nodes should be followed as in critical organ group. Further radiobiological studies should be done to find the dose constraints for the axillary space and internal mammary chain.

\section{References}

1. Habermann EB, Abbott A, Parsons HM, Virnig BA, Al-Refaie WB, et al (2010) Are mastectomy rates really increasing in the United States? J Clin Oncol 28: 3437-3441.

2. El Saghir NS, Khalil MK, Eid T, El Kinge AR, Charafeddine M, et al. (2007) Trends in epidemiology and management of breast cancer in developing Arab countries: a literature and registry analysis. Int J Surg 5: 225-233.

3. Overgaard M, Nielsen HM, Overgaard J (2007) Is the benefit of postmastectomy irradiation limited to patients with four or more positive nodes, as recommended in international consensus reports? A subgroup analysis of the DBCG 82 b\&c randomized trials. Radiother Oncol 82: 247-253.

4. Clarke M, Collins R, Darby S, Davies C, Elphinstone P, et al. (2005) Effects of radiotherapy and of differences in the extent of surgery for early breast cancer on local recurrence and 15-year survival: an overview of the randomised trials. Lancet 366:2087-2106.

5. Larson D, Weinstein M, Goldberg I, Silver B, Recht A, et al. (1986) Edema of the arm as a function of the extent of axillary surgery in the patients with stage I-II carcinoma of the breast treated with primary radiotherapy. Int J Radiat Oncol Biol Phys 12:1575-1582.

6. Beckham WA, Popescu CC, Patenaude VV, Wai ES, Olivotto IA (2007) Is multibeam IMRT better than standard treatment for patients with leftsided breast cancer? Int J Radiat Oncol Biol Phys 69: 918-924.

7. Dogan N, Cuttino L, Lloyd R, Bump EA, Arthur DW (2007) Optimized dose coverage of regional lymph nodes in breast cancer: the role of intensity-modulated radiotherapy. Int J Radiat Oncol Biol Phys 68: 1238-1250.

8. Cavey ML, Bayouth JE, Endres EJ, Pena JM, Colman M, et al. (2005) Dosimetric comparison of conventional and forward-planned intensitymodulated techniques for comprehensive locoregional irradiation of postmastectomy left breast cancers. Med Dosim 30:107-116.

9. Gauer T, Engel K, Kiesel A, Albers D, Rades D (2010) Comparison of electron IMRT to helical photon IMRT and conventional photon irradiation for treatment of breast and chest wall tumours. Radiother Oncol 94: 313-18. 
Citation: Govardhan HB, Thimmaiah N, Khaleel IA, Pradhan S, jain R, et al. (2017) Comparison of Incidental Radiation Dose to Axilla and Internal Mammary Nodal Area by Conventional, 3DCRT, and IMRT Technique in Carcinoma of Breast. J Nucl Med Radiat Ther 8: 325. doi: $10.4172 / 2155-9619.1000325$

Page 6 of 6

10. Holland R, Veling SH, Mravunac M, Hendriks JH (1985) Histologic multifocality of Tis, T1-2 breast carcinomas. Implications for clinical trials of breast-conserving surgery. Cancer 56: 979-990.

11. Fisher B, Redmond C, Poisson R, Margolese R, Wolmark N, et al. (1989) Eight-year results of a randomized clinical trial comparing total mastectomy and lumpectomy with or without irradiation in the treatment of breast cancer. N Engl J Med 320: 822-828.

12. van den Hoven I, Voogd AC, Roumen RM (2015) A paradigm shift in axillary breast cancer treatment; From "Treat All-Except," toward "Treat None-Unless". Clin Breast Cancer 15: 399-402.

13. Petrek J, Lerner R (2000) Lymphedema. In: Disease of breast. 2nd ed. Philadelphia: Lippincott, Williams \& Wilkins, USA. pp: 1033-1039.

14. Fraile M, Rull M, Julián FJ, Fusté F, Barnadas A, et al. (2000) Sentinel node biopsy as a practical alternative to axillary lymph node dissection in breast cancer patients: an approach to its validity. Ann Oncol 11: 701-705

15. RTOG breast cancer atlas for radiation planning: consensus definition.

16. Cady B, Stone MD, Schuler JG, Thakur R, Wanner MA, et al. (1996) The new era in breast cancer. Invasion, size, and nodal involvement dramatically decreasing as a result of mammographic screening. Arch Surg 131: 301-308.

17. Hong L, Hunt M, Chui C, Spirou S, Forster K, et al. (1999) Intensitymodulated tangential beam irradiation of the intact breast. Int J Radiat Oncol Biol Phys 44: 1155-1164.

18. Krasin M, McCall A, King S, Olson R, Emami B (2000) Evaluation of a standard breast tangent technique: a dose-volume analysis of tangential irradiation using three dimensional tools. Int J Radiat Oncol Biol Phys 47: 327-333.

19. Alco G, Igdem SI, Ercan T, Dincer M, Senturk R, et al. (2010) Coverage of axillary lymph nodes with high tangential fields in breast radiotherapy. $\mathrm{Br}$ J Radiol 83: 1072-1076.

20. Li XA, Tai A, Arthur DW, Buchholz TA, Macdonald S, et al. (2009) Variability of target and normal structure delineation for breast cancer radiotherapy: an RTOG Multi-Institutional and Multiobserver Study. Int J Radiat Oncol Biol Phys 73: 944-951.

21. Orecchia R, Huscher A, Leonardi MC, Gennari R, Galimberti V, et al. (2005) Irradiation with standard tangential breast fields in patients treated with conservative surgery and sentinel node biopsy: using a threedimensional tool to evaluate the first level coverage of the axillary nodes. Br J Radiol 78: 51-4.

22. Aristei C, Chionne F, Marsella AR, Alessandro M, Rull A, et al. (2001) Evaluation of level I and II axillary nodes included in the standard breast tangential fields and calculation of the administered dose: results of a prospective study. Int J Radiat Oncol Biol Phys 51: 69-73.

23. Smitt MC, Goffinet DR (1999) Utility of three-dimensional planning for axillary node coverage with breast-conserving radiation therapy: early experience. Radiology 210: 221-26.

24. Goodman RL, Grann A, Saracco P, Needham MF (2001) The relationship between radiation fields and regional lymph nodes in carcinoma of the breast. Int J Radiat Oncol Biol Phys 50: 99-105.

25. Ribeiro GG, Dunn G, Swindell R, Harris M, Banerjee SS (1990) Conservation of the breast using two different radiotherapy techniques: interim report of a clinical trial. Clin Oncol 2: 27-34.

26. Chu KU, Turner RR, Hansen NM, Brennan MB, Bilchik A, et al. (1999) Do all patients with sentinel node metastasis from breast carcinoma need complete axillary node dissection? Ann Surg 229: 536-541.

27. Withers HR, Peters LJ, Taylor JM (1995) Dose-response relationship for radiation therapy of subclinical disease. Int J Radiat Oncol Biol Phys 31: 353-359.

28. Suwinski R, Withers HR (2003) Time factor and treatment strategies in subclinical disease. Int J Radiat Biol 79: 495-502.

29. Withers HR, Suwinski R (1998) Radiation dose response for subclinical metastases. Semin Radiat Oncol 8: 224-228.

30. Lin PP, Allison DC, Wainstock J, Miller KD, Dooley WC, et al. (1993) Impact of axillary lymph node dissection on the therapy of breast cancer patients. J Clin Oncol 11: 1536-1544.

31. Mansel RE, Fallowfield L, Kissin M, Goyal A, Newcombe RG, et al. (2006) Randomized multicenter trial of sentinel node biopsy versus standard axillary treatment in operable breast cancer: the ALMANAC Trial. J Natl Cancer Inst 98:599-609. 